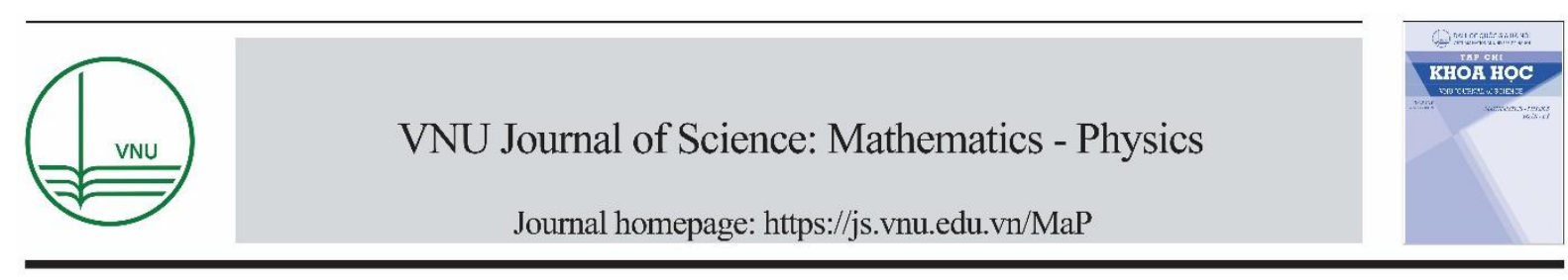

Original Article

\title{
On Stability for Hybrid System under Stochastic Perturbations
}

\author{
Cao Tan Binh ${ }^{1, *}$, Ta Cong Son ${ }^{2}$ \\ ${ }^{1}$ Quy Nhon University, 170 An Duong Vuong, Quy Nhon, Vietnam \\ ${ }^{2}$ VNU University of Science, 334 Nguyen Trai, Hanoi, Vietnam
}

Received 13 May 2020

Revised 22 July 2020; Accepted 30 August 2020

\begin{abstract}
The aim of this paper is to find out suitable conditions for almost surely exponential stability of communication protocols, considered for nonlinear hybrid system under stochastic perturbations. By using the Lyapunov-type function, we proved that the almost surely exponential stability remain be guaranteed as long as a bound on the maximum allowable transfer interval (MATI) is satisfied.

Keywords: Networked Control System, almost surely exponential stability, maximum allowable transfer interval, Lyapunov function.
\end{abstract}

\section{Introduction}

In recent years, Networked Control Systems (NCS) were addressed strongly in the control community because of its extensive applications in wireless as well as wireline. The pioneering papers were proposed by Walsh, Beldiman and Bushnell [10,11, 12]. They introduced about stability of control systems with deterministic protocol. More recently, quite many articles and literatures referred to study stability of hybrid systems by specifically showing the Lyapunov-type function and bounds on the maximum allowable transfer interval (MATI), see [1, 2, 3, 4, 8, 6, 9, 13] for more details. This paper is divided into two sections. Beside Introduction, we state Preliminary and main problem in the second section.

\footnotetext{
${ }^{*}$ Corresponding author.

Email address: caotanbinh@qnu.edu.vn
}

https//doi.org/ 10.25073/2588-1124/vnumap.4522 
In [5], the authors solved entirely for researching the stable types of solution of hybrid systems, modelled as follows:

$$
\begin{aligned}
& \dot{x}(t)=f(x(t), e(t)), t \in\left(t_{k}, t_{k+1}\right), \\
& \dot{e}(t)=g(x(t), e(t)), t \in\left(t_{k}, t_{k+1}\right), \\
& \dot{\tau}(t)=1, t \in\left(t_{k}, t_{k+1}\right), \\
& \tau\left(t_{k}^{+}\right)=0, \\
& x\left(t_{k}^{+}\right)=x\left(t_{k}\right), \\
& e\left(t_{k}^{+}\right)=b_{k} h\left(k, e\left(t_{k}\right)\right)+\left(1-b_{k}\right) e\left(t_{k}\right), k=0,1,2, \ldots
\end{aligned}
$$

Remind here that the variable $b_{k}$ belongs to the set $\{0,1\}$. If $b_{k}=1$ then transmission is successful, and the protocol $h$ determines the updated error. While if $b_{k}=0$ then the error remains unchanged at the $t_{k}$. We get a sequence $\left(b_{k}\right)_{k \in \square}$. Let $S:=\{0,1\}$ and the probability space $\left(S^{\square}, F_{b}, P\right)$ with the sequence space

$$
S^{\square}:=\left\{\left(b_{k}\right)_{k \in \square}: b_{k} \in S, \forall k \in \square\right\}
$$

where the $\sigma$-algebra $F_{b}:=2^{S} \times 2^{S} \times \ldots$ and the probability $P$ satisfying

$$
P\left(b \in S^{\square}: b_{k}=1\right)=p, \forall k \in \square .
$$

We also assume that the random variables $b_{k}$ are independently and identically distributed.

Motivated from this paper, we concern to hybrid system in which exogenously stochastic perturbation is a Wiener process. This is, up to now, one of proposed problems remain have not been solved yet. To solve the problem, we make use of tools as introduced in [5] by defining $\tau_{\text {MATI }}$ or choosing the Lyapunov function $W$ for protocol. We also, of course, use other tools for stochastic stability from [7] in order to support our proof.

\section{Preliminary and main result}

Let us now consider the perturbed hybrid system that is of form

$$
\begin{aligned}
& d x(t)=f_{1}(x(t), e(t)) d t+f_{2}(x(t), e(t)) d \mathrm{w}(t), t \in\left(t_{k}, t_{k+1}\right), \\
& d e(t)=g_{1}(x(t), e(t)) d t+g_{2}(x(t), e(t)) d \mathrm{w}(t), t \in\left(t_{k}, t_{k+1}\right), \\
& \dot{\tau}(t)=1, t \in\left(t_{k}, t_{k+1}\right), \\
& \tau\left(t_{k}^{+}\right)=0, \\
& x\left(t_{k}^{+}\right)=x\left(t_{k}\right), \\
& e\left(t_{k}^{+}\right)=b_{k} h\left(k, e\left(t_{k}\right)\right)+\left(1-b_{k}\right) e\left(t_{k}\right), k=0,1,2, \ldots
\end{aligned}
$$

where $x \in \square^{n}$ is the state of the system, $e \in \square^{n}$ is the error at the controller, $h$ is the update function that models the particular protocol, $\tau$ is a timer to constrain both the transmission interval and the 
transmission delay, and $w(t)$ is a Wiener process. In this paper, suppose that $f_{1}, f_{2}, g_{1}$ and $g_{2}$ satisfy Lipschitz and linear growth conditions which guarantee the existence and uniqueness of the solution of (2). Assume furthermore that $f_{1}(0,0)=f_{2}(0,0)=g_{1}(0,0)=g_{2}(0,0)$ and $h(k, 0)=0$ for all $k \in \square$. So system (2) has the solution $\xi(t):=(x(t)$; $e(t))=(0,0)$ corresponding to the initial value $\xi^{*}:=\left(x^{*}, e^{*}\right)=(0,0)$.

Now, we introduce the concept of almost surely exponential stability, which can be found in Mao [7].

Definition 1 Consider the system (2). The solution $\xi^{*}=\left(x^{*}, e^{*}\right)=(0,0)$ of $(1)$ is called almost surely exponentially stable, if for all $\xi_{0}$

$$
\limsup _{t \rightarrow \infty} \frac{1}{t} \log \left\|\xi\left(t, 0, \xi_{0}, b\right)\right\|<0, \text { almost surely. }
$$

We need the following assumptions for the stability of network and system.

Assumption (A1) The probability $\mathrm{p} \in(0,1)$ of successful transmission of the $k$-th sampling time is identical for all $\mathrm{k} \in \square$ and independent of $\mathrm{k} \in \square$.

Assumption (A2) The stochastic perturbations $b$ and $w$ are mutually independent. Put $F_{b}$ is the $\sigma$-algebra generated by $\left(b_{k}\right)_{k \in \square}$, and $F_{\mathrm{w}}$ is the $\sigma$-algebra generated by $\{\mathrm{w}(\mathrm{t})\}_{t \geq 0}$. The system (2) defined on a probability space $(\Omega, F, P)$ where $F=\sigma\left\{F_{b} \cup F_{\mathrm{w}}\right\}$. Hereafter, we use notation $E_{b}($. instead of $E_{b}\left(. \mid F_{\mathrm{b}}\right)$ and $E_{\mathrm{w}}($.$) instead of E_{\mathrm{w}}\left(. \mid F_{\mathrm{w}}\right)$.

Assumption (A3) Lyapunov functions for the protocol and the perturbed system.

(i) There exist constants $0<a_{1}, a_{2}, 0<\lambda<1$ such that for all $e \in \square^{n}$ :

$$
\begin{gathered}
a_{1}\|e\|^{2} \leq \mathrm{W}(\mathrm{e}) \leq \mathrm{a}_{2}\|e\|^{2} \\
W(h(k, e)) \leq \lambda W(e) .
\end{gathered}
$$

(ii) The evolution of Lyapunov function $W$ is bounded in the sense that there exist a constant $\alpha \geq 0, \beta \in \square$ and a continuous function $H: \square^{n} \rightarrow \square+$ such that for all $x, e \in \square^{n}$ :

$$
\frac{\partial W}{\partial e} \cdot g_{1}(x, e)=\left\langle\frac{\partial W}{\partial e}, g_{1}^{T}(x, e)\right\rangle \leq 2 \alpha W(e)+\beta H(x)
$$

(iii) There exist a $C^{2}$ Lyapunov function $V$ and constants $b_{1}, b_{2}, b_{3}>0$ such that for all $x, e \in \square^{n}$

$$
\begin{gathered}
b_{1}\|x\|^{2} \leq V(x) \leq b_{2}\|x\|^{2} \\
L V(x):=\frac{\partial V}{\partial x} \cdot f_{1}(x, e)+\frac{1}{2} f_{2}^{T}(x, e) \cdot \frac{\partial^{2} V}{\partial x^{2}} \cdot f_{2}(x, e) \leq-b_{3} V(x),
\end{gathered}
$$

where 


$$
\begin{gathered}
f_{i}^{T}(x, e)=\left[f_{i}^{(1)} \cdots f_{i}^{(n)}\right] \text { is the transpose of } f_{i}(x, e) \in \square^{n}, i=1,2 \\
g_{i}^{T}(x, e)=\left[g_{i}^{(1)} \cdots g_{i}^{(n)}\right] \text { is the transpose of } g_{i}(x, e) \in \square^{n}, i=1,2 \\
\frac{\partial^{2} V}{\partial x^{2}}=V_{x x}:=\left[\begin{array}{ccc}
\frac{\partial^{2} V}{\partial x_{1} \partial x_{1}} & \cdots & \frac{\partial^{2} V}{\partial x_{1} \partial x_{n}} \\
\vdots & \ddots & \vdots \\
\frac{\partial^{2} V}{\partial x_{n} \partial x_{1}} & \cdots & \frac{\partial^{2} V}{\partial x_{n} \partial x_{n}}
\end{array}\right]_{n \times n}, \frac{\partial^{2} V}{\partial x \partial e}=\frac{\partial^{2} V}{\partial e \partial x}:=\left[\begin{array}{ccc}
\frac{\partial^{2} V}{\partial x_{1} \partial e_{1}} & \cdots & \frac{\partial^{2} V}{\partial x_{1} \partial e_{n}} \\
\vdots & \ddots & \vdots \\
\frac{\partial^{2} V}{\partial x_{n} \partial e_{1}} & \cdots & \frac{\partial^{2} V}{\partial x_{n} \partial e_{n}}
\end{array}\right]_{n \times n} \\
\frac{\partial V}{\partial x}=\left[\frac{\partial V}{\partial x_{1}} \cdots \frac{\partial V}{\partial x_{n}}\right], \frac{\partial V}{\partial x} \cdot f_{1}(x, e)=\left\langle\frac{\partial V}{\partial x}, f_{1}^{T}(x, e)\right\rangle, \frac{\partial V}{\partial e} \cdot g_{1}(x, e)=\left\langle\frac{\partial V}{\partial e}, g_{1}^{T}(x, e)\right\rangle .
\end{gathered}
$$

Here, $\tau_{\text {MATI }}$ follows from the equation

$$
\dot{\phi}=-2 \alpha \phi-\gamma\left(\phi^{2}+1\right), \phi(0)=\eta^{-1} .
$$

We choose $\tau(\eta)$ such that for all $\tau \in[0, \tau(\eta)]$ we have

$$
\phi_{\eta}(\tau) \in\left[\eta, \eta^{-1}\right]
$$

see [5] for more details.

Theorem 2 Consider the system (2). Assume that (A1), (A2) and (A3) hold. If there exist $\eta \in(0,1)$ and $\gamma>0$ as defined in (8) satisfying

$$
g_{2}^{T}(x, e) \cdot \frac{\partial^{2} W}{\partial e^{2}} \cdot g_{2}(x, e) \leq 2\left[\left(2 \gamma \eta-b_{3}\right) W(e)-\beta H(x)\right] \text { for almost all } x, e \in \square^{n}
$$

then the solution $\xi^{*}=(0,0)$ of system (2) is almost surely exponentially stable.

Proof: We first assume that system (2a), (2b) is almost surely exponentially stable. Consider Lyapunov-type function

$$
U(\xi, \tau)=U(x, e, \tau):=V(x)+\gamma \phi(\tau) W(e) .
$$

It follows that

$$
b_{1}\|x\|^{2} \leq V(x) \leq b_{2}\|x\|^{2}, a_{1}\|e\|^{2} \leq \mathrm{W}(\mathrm{e}) \leq \mathrm{a}_{2}\|e\|^{2}, \eta \leq \phi(\tau) \leq \frac{1}{\eta} .
$$

We yield

$$
b_{1}\|x\|^{2}+\gamma \eta a_{1}\|e\|^{2} \leq U(x, e, \tau)=V(x)+\gamma \phi(\tau) \mathrm{W}(\mathrm{e}) \leq b_{2}\|x\|^{2}+\gamma \eta^{-1} a_{2}\|e\|^{2}
$$

and

$$
m\|\xi\|^{2}=m\|(x, e)\|^{2} \leq U(x, e, \tau) \leq M\|(x, e)\|^{2}=M\|\xi\|^{2}
$$

where $m=\min \left\{b_{1}, \gamma \eta a_{1}\right\}, M=\max \left\{b_{2}, \gamma \eta^{-1} a_{2}\right\}$.

By Ito's formula and Assumption (A3), we can derive that 


$$
\begin{aligned}
d U(x, e, \tau)= & \frac{\partial U}{\partial x} d x+\frac{\partial U}{\partial e} d e+\frac{\partial U}{\partial \tau} d \tau+\frac{1}{2}\left[f_{2}^{T}(x, e) \cdot \frac{\partial^{2} U}{\partial x^{2}} \cdot f_{2}(x, e)+g_{2}^{T}(x, e) \cdot \frac{\partial^{2} U}{\partial e^{2}} \cdot g_{2}(x, e)\right] d t \\
= & \frac{\partial V}{\partial x}\left[f_{1}(x, e) d t+f_{2}(x, e) d w\right]+\gamma \phi(\tau) \frac{\partial W}{\partial e}\left[g_{1}(x, e) d t+g_{2}(x, e) d w\right] \\
& +\gamma \dot{\phi}(\tau) W(e) \dot{\tau} d t+\frac{1}{2}\left[f_{2}^{T}(x, e) \cdot \frac{\partial^{2} V}{\partial x^{2}} \cdot f_{2}(x, e)+\gamma \phi(\tau) g_{2}^{T}(x, e) \cdot \frac{\partial^{2} W}{\partial e^{2}} \cdot g_{2}(x, e)\right] d t \\
= & L V(x) d t+\left[\gamma \phi(\tau) \frac{\partial W}{\partial e} \cdot g_{1}(x, e)+\gamma \dot{\phi}(\tau) W(e)+\frac{1}{2} \gamma \phi(\tau) g_{2}^{T}(x, e) \cdot \frac{\partial^{2} W}{\partial e^{2}} \cdot g_{2}(x, e)\right] d t \\
& +\left[\frac{\partial V}{\partial x} \cdot f_{2}(x, e)+\gamma \phi(\tau) \frac{\partial W}{\partial e} \cdot g_{2}(x, e)\right] d w
\end{aligned}
$$

and

$$
\begin{aligned}
\gamma \phi(\tau) & \frac{\partial W}{\partial e} \cdot g_{1}(x, e)+\gamma \dot{\phi}(\tau) W(e)+\frac{1}{2} \gamma \phi(\tau) g_{2}^{T}(x, e) \cdot \frac{\partial^{2} W}{\partial e^{2}} \cdot g_{2}(x, e) \\
= & \gamma \phi(\tau) \frac{\partial W}{\partial e} \cdot g_{1}(x, e)+\gamma\left[-2 \alpha \phi(\tau)-\gamma\left(\phi^{2}(\tau)+1\right)\right] W(e)+\frac{1}{2} \gamma \phi(\tau) g_{2}^{T}(x, e) \cdot \frac{\partial^{2} W}{\partial e^{2}} \cdot g_{2}(x, e) \\
\stackrel{(5),(8),(10)}{\leq} & \gamma \phi(\tau)[2 \alpha W(e)+\beta H(x)]-2 \gamma \alpha \phi(\tau) W(e)-\gamma^{2}\left[\phi^{2}(\tau)+1\right] W(e) \\
& +\gamma \phi(\tau)\left[\left(2 \gamma \eta-b_{3}\right) W(e)-\beta H(x)\right] \\
= & 2 \gamma \alpha \phi(\tau) W(e)+\gamma \beta \phi(\tau) H(x)-2 \gamma \alpha \phi(\tau) W(e)-\gamma^{2} \phi^{2}(\tau) W(e)-\gamma^{2} W(e) \\
& +\gamma \phi(\tau)\left[\left(\gamma \eta+\gamma \eta-b_{3}\right) W(e)-\beta H(x)\right] \\
\stackrel{(9)}{\leq} & -\gamma \phi(\tau) b_{3} W(e) .
\end{aligned}
$$

Therefore

$$
\begin{aligned}
d U(x, e, \tau) \stackrel{(7),(14)}{\leq}-b_{3} V(x) d t-\gamma \phi(\tau) b_{3} W(e) d t+\left[\frac{\partial V}{\partial x} \cdot f_{2}(x, e)+\gamma \phi(\tau) \frac{\partial W}{\partial e} \cdot g_{2}(x, e)\right] d w \\
=-b_{3} U(x, e, \tau) d t+\left[\frac{\partial V}{\partial x} \cdot f_{2}(x, e)+\gamma \phi(\tau) \frac{\partial W}{\partial e} \cdot g_{2}(x, e)\right] d w
\end{aligned}
$$

This implies

$$
d E_{w}[U(x, e, \tau)] \leq-b_{3} E_{w}[U(x, e, \tau)] d t .
$$

For each $k=1,2, \ldots$, integrating both sides of (16) from $t_{k-1}^{+}$to $t_{k}$, we get

$$
\begin{aligned}
E_{w}\left[U\left(x\left(t_{k}, b\right), e\left(t_{k}, b\right), \tau\left(t_{k}\right)\right)\right] & \leq E_{w}\left[U\left(x\left(t_{k-1}^{+}, b\right), e\left(t_{k-1}^{+}, b\right), \tau\left(t_{k-1}^{+}\right)\right)\right]+\int_{t_{k-1}^{+}}^{t_{k}} E_{w}\left[-b_{3} U(x, e, \tau)\right] d t \\
& \leq E_{w}\left[U\left(x\left(t_{k-1}^{+}, b\right), e\left(t_{k-1}^{+}, b\right), \tau\left(t_{k-1}^{+}\right)\right)\right] .
\end{aligned}
$$

If at time $t_{k}$ transmission is successful, i.e. if $b_{k}=1$, then

$$
U\left(x\left(t_{k}^{+}, b\right), e\left(t_{k}^{+}, b\right), \tau\left(t_{k}^{+}\right)\right) \leq V\left(x\left(t_{k}, b\right)\right)+\eta^{-2} \lambda \gamma \phi\left(\tau\left(t_{k}\right)\right) W\left(e\left(t_{k}, b\right)\right) .
$$


On the other hand if transmission fails, i.e. if $b_{k}=0$ then

$$
U\left(x\left(t_{k}^{+}, b\right), e\left(t_{k}^{+}, b\right), \tau\left(t_{k}^{+}\right)\right) \leq V\left(x\left(t_{k}, b\right)\right)+\eta^{-2} \gamma \phi\left(\tau\left(t_{k}\right)\right) W\left(e\left(t_{k}, b\right)\right) .
$$

These give

$$
\begin{aligned}
& E_{b}\left\{E_{w}\left[U\left(x\left(t_{k}^{+}, b\right), e\left(t_{k}^{+}, b\right), \tau\left(t_{k}^{+}\right)\right) \mid\left(x\left(t_{k}^{+}, b\right), e\left(t_{k}^{+}, b\right)\right)\right]\right\} \\
& \leq p\left\{E_{w}\left[V\left(x\left(t_{k}, b\right)\right)\right]+\eta^{-2} \lambda \gamma \phi\left(\tau\left(t_{k}\right)\right) E_{w}\left[W\left(e\left(t_{k}, b\right)\right)\right]\right\} \\
& +(1-p)\left\{E_{w}\left[V\left(x\left(t_{k}, b\right)\right)\right]+\eta^{-2} \gamma \phi\left(\tau\left(t_{k}\right)\right) E_{w}\left[W\left(e\left(t_{k}, b\right)\right)\right]\right\} \\
& <E_{w}\left[U\left(x\left(t_{k}, b\right), e\left(t_{k}, b\right), \tau\left(t_{k}\right)\right)\right]-\kappa \gamma \eta E_{w}\left[W\left(e\left(t_{k}, b\right)\right)\right]
\end{aligned}
$$

where $\kappa:=1-(1-p+p \lambda) \eta^{-2}$.

From (16) it follows that

$$
E_{w}[U(\xi(t, b), \tau(t))]=E_{w}[U(x(t, b), e(t, b), \tau(t))]<e^{-b_{3}\left(t-t_{k}\right)} E_{w}\left[U\left(\xi\left(t_{k}^{+}, b\right), \tau\left(t_{k}^{+}\right)\right)\right] .
$$

Taking expectation in $b$, we obtain

$$
e^{b_{3} t} E_{b}\left\{E_{w}[U(\xi(t, b), \tau(t))]\right\}<e^{b_{3} t_{k}} E_{b}\left\{E_{w}\left[U\left(\xi\left(t_{k}^{+}, b\right), \tau\left(t_{k}^{+}\right)\right)\right]\right\}
$$

and

$$
\begin{aligned}
& 0 \leq e^{b_{3} t_{k}} E_{b}\left\{E_{w}\left[U\left(\xi\left(t_{k}^{+}, b\right), \tau\left(t_{k}^{+}\right)\right)\right]\right\}=e^{b_{3} t_{k}} E_{b}\left\{E_{w}\left[U\left(\xi\left(t_{k}^{+}, b\right), \tau\left(t_{k}^{+}\right)\right) \mid \xi\left(t_{k}, b\right)\right]\right\} \\
& \stackrel{(17),(18)}{\leq} M E_{w}\left\|\xi_{0}\right\|^{2}-\kappa \gamma \eta \sum_{i=0}^{k} e^{b_{3} t_{i}} E_{b}\left\{E_{w}\left[W\left(e\left(t_{i}, b\right)\right)\right]\right\} .
\end{aligned}
$$

From (12), (19) and (20), it follows that

$$
\begin{aligned}
m e^{b_{3} t} E_{b}\left\{E_{w}\|\xi(t, b)\|\right\} & \leq e^{b_{3} t} E_{b}\left\{E_{w}[U(\xi(t, b), \tau(t))]\right\} \\
& <e^{b_{3} t_{k}} E_{b}\left\{E_{w}\left[U\left(\xi\left(t_{k}^{+}, b\right), \tau\left(t_{k}^{+}\right)\right)\right]\right\} \leq M E_{w}\left\|\xi_{0}\right\|^{2} .
\end{aligned}
$$

Hence

$$
E_{b}\left\{E_{w}\|\xi(t, b)\|\right\} \leq \frac{M}{m} E_{w}\left\|\xi_{0}\right\|^{2} e^{-b_{3} t}, \forall t \geq 0
$$

From the system (2), we have

$$
x(t)=x\left(t_{k}\right)+\int_{t_{k}}^{t} f_{1} d s+\int_{t_{k}}^{t} f_{2} d w(s)
$$

and

$$
e(t)=e\left(t_{k}\right)+\int_{t_{k}}^{t} g_{1} d s+\int_{t_{k}}^{t} g_{2} d w(s) .
$$

In addition, the conditions $f_{1}(0,0)=f_{2}(0,0)=g_{1}(0,0)=g_{2}(0,0)$ lead to exist a positive constant $K$ such that

$$
\left\{\begin{array}{l}
\left\|f_{1}(x, e)\right\|^{2} \vee\left\|f_{2}(x, e)\right\|^{2} \leq K\|(x, e)\|^{2} \\
\left\|g_{1}(x, e)\right\|^{2} \vee\left\|g_{2}(x, e)\right\|^{2} \leq K\|(x, e)\|^{2}
\end{array}\right.
$$


Therefore, we obtain

$$
\begin{aligned}
E_{w}\|x(t)\|^{2} & \leq 3\left[E_{w} x^{2}\left(t_{k}\right)+E_{w}\left(\int_{t_{k}}^{t} f_{1} d s\right)^{2}+E_{w}\left(\int_{t_{k}}^{t} f_{2} d w(s)\right)^{2}\right] \\
& \leq 3\left[E_{w} x^{2}\left(t_{k}\right)+\left(t-t_{k}\right) \int_{t_{k}}^{t} E_{w} f_{1}^{2} d s+\int_{t_{k}}^{t} E_{w} f_{2}^{2} d s\right] \\
& \stackrel{(22)}{\leq}\left[E_{w} x^{2}\left(t_{k}\right)+\bar{\tau} K \int_{t_{k}}^{t} E_{w}\|\xi(s)\|^{2} d s+K \int_{t_{k}}^{t} E_{w}\|\xi(s)\|^{2} d s\right] \\
& \leq 3\left[E_{w} x^{2}\left(t_{k}\right)+(\bar{\tau}+1) K \int_{t_{k}}^{t} E_{w}\|\xi(s)\|^{2} d s\right]
\end{aligned}
$$

and

$$
E_{w}\|e(t)\|^{2} \leq 3\left[E_{w} e^{2}\left(t_{k}\right)+(\bar{\tau}+1) K \int_{t_{k}}^{t} E_{w}\|\xi(s)\|^{2} d s\right]
$$

As a result

$$
E_{w}\|\xi\|^{2}=E_{w}\|(x, e)\|^{2}=E_{w}\|x(t)\|^{2}+E_{w}\|e(t)\|^{2} \leq 3\left[E_{w}\left\|\xi\left(t_{k}\right)\right\|^{2}+2(\bar{\tau}+1) K \int_{t_{k}}^{t} E_{w}\|\xi(s)\|^{2} d s\right] .
$$

Hence

$$
\begin{aligned}
E_{b}\left(\sup _{t_{k} \leq t \leq t_{k+1}} E_{w}\|\xi(t, b)\|^{2}\right) & \leq 3\left[E_{b} E_{w}\left\|\xi\left(t_{k}, b\right)\right\|^{2}+2(\bar{\tau}+1) K \int_{t_{k}}^{t_{k+1}} E_{b} E_{w}\|\xi(s, b)\|^{2} d s\right] \\
& \leq 3\left[\frac{M}{m} E_{w}\left\|\xi_{0}\right\|^{2} e^{-b_{3} t_{k}}+2(\bar{\tau}+1) K \int_{t_{k}}^{t_{k+1}} E_{w}\left\|\xi_{0}\right\|^{2} e^{-b_{3} s} d s\right] \\
& \leq 3\left[1-\frac{2}{b_{3}} K(\bar{\tau}+1)\left(1-e^{b_{3} \bar{\tau}}\right)\right] \frac{M}{m} E_{w}\left\|\xi_{0}\right\|^{2} e^{-b_{3} t_{k+1}} \\
& \leq C e^{-b_{3} t_{k+1}},
\end{aligned}
$$

where

$$
C=3\left[1-\frac{2}{b_{3}} K(\bar{\tau}+1)\left(1-e^{b_{3} \bar{\tau}}\right)\right] \frac{M}{m} E_{w}\left\|\xi_{0}\right\|^{2} .
$$

Applying Chebyshev's inequality, we get

$$
\begin{aligned}
P\left(b: \sup _{t_{k} \leq t \leq t_{k+1}} E_{w}\|\xi(t, b)\|^{2}>e^{-\frac{b_{3}}{2} t_{k+1}}\right) & \leq \frac{E_{b}\left(\sup _{t_{k} \leq t \leq t_{k+1}} E_{w}\|\xi(t, b)\|^{2}\right)}{e^{-\frac{b_{3}}{2} t_{k+1}}} \\
& \leq C e^{-\frac{b_{3} t_{k+1}}{2^{2}} .}
\end{aligned}
$$

Since $t_{0}=0$ and $0<\delta<t_{k+1}-t_{k} \leq \bar{\tau}$, it is clear that

$$
\begin{aligned}
\sum_{k=0}^{\infty} e^{-\frac{b_{3}}{2} t_{k+1}} & =e^{-\frac{b_{3}}{2} t_{1}}+e^{-\frac{b_{3}}{2} t_{2}}+\cdots+ \\
& =e^{-\frac{b_{3}}{2}\left(t_{1}-t_{0}\right)}+e^{-\frac{b_{3}}{2}\left(t_{2}-t_{1}+t_{1}-t_{0}\right)}+\cdots<+\infty .
\end{aligned}
$$


Using Borel-Cantelli's lemma argument (see Mao [7]) to conclude that there exist a set $\Omega_{1}$ with $P\left(\Omega_{1}\right)=1$ and an integer-value random variable $k_{0}$ such that for every $b \in \Omega_{1}$ we have

$$
\sup _{t_{k} \leq t \leq t_{k+1}} E_{w}\|\xi(t, b)\|^{2} \leq e^{-\frac{b_{3}}{2} t_{k+1}}, \forall k \geq k_{0}(b) .
$$

That means

$$
E_{w}\|\xi(t, b)\|^{2} \leq e^{-\frac{b_{3}}{2} t_{k+1}}, \forall t \in\left(t_{k}, t_{k+1}\right), \forall k \geq k_{0}(b)
$$

Similarly to argument as above, using Borel-Cantelli's lemma again, there exist a set $\Omega_{2}$ with $P\left(\Omega_{2}\right)=1$ and an integer-value random variable $k_{1}$ such that for every $w \in \Omega_{2}$ we have

$$
\|\xi(t, b)\|^{2} \leq e^{-\frac{b_{3}}{2} k_{k+1}}, \forall t \in\left(t_{k}, t_{k+1}\right), \forall k \geq k_{1}(w) .
$$

Let $k_{c}=\max \left\{k_{0}, k_{1}\right\}, \Omega_{0}=\Omega_{1} \cap \Omega_{2}$, we have $P\left(\Omega_{0}\right)=1$ and

$$
\|\xi(t, b)\|^{2} \leq e^{-\frac{b_{3}}{2} t_{k+1}}, \forall t \in\left(t_{k}, t_{k+1}\right), \forall k \geq k_{c}(w),(b, w) \in \Omega_{0} .
$$

Consequently

$$
\limsup _{t \rightarrow \infty} \frac{1}{t} \log \|(\xi(t), b)\| \leq-\frac{b_{3}}{8}<0
$$

The proof is completed.

Remark 3 The inequalities (5) and (10) are existent. In fact, we choose $g_{1}(x, e)=g_{2}(x, e)=e$, $W(e)=\|e\|=\left(e_{1}^{2}+e_{2}^{2}\right)^{1 / 2}$ and $\beta=0$. Then we have

$$
\frac{\partial W}{\partial e} \cdot g_{1}(x, e)=\left\langle\frac{\partial W}{\partial e}, g_{1}^{T}(x, e)\right\rangle=\left(e_{1}^{2}+e_{2}^{2}\right)^{1 / 2} \leq 2 \alpha W(e), \forall \alpha \geq 0,
$$

Moreover,

$$
g_{2}^{T} \cdot \frac{\partial^{2} W(e)}{\partial e^{2}} \cdot g_{2}=0 \leq 2\left(2 \gamma \eta-b_{3}\right) W(e)
$$

as long as $2 \gamma \eta-b_{3} \geq 0$.

\section{References}

[1] D. Carnevale, A. R. Teel, D. Nesic, Further results on stability of networked control systems: a lyapunov approach, In 2007 American Control Conference, IEEE. (2007) 1741-1746.

[2] D. Carnevale, A. R. Teel, D. Nesic, A Lyapunov proof of an improved maximum allowable transfer interval for networked control systems, IEEE Transactions on Automatic Control. 52 (2007) 892-897.

[3] D. Christmann, On the behavior of black bursts in tick-synchronized networks, Techn. Ber. 337 (2010). 
[4] M. B. Cloosterman, N. Van de Wouw, W. P. M. H. Heemels, H. Nijmeijer, Stability of networked control systems with uncertain time-varying delays, IEEE Transactions on Automatic Controll. 54 (2009) 1575-1580.

[5] L. H. Duc, D. Christmann, R. Gotzhein, S. Siegmund, F. Wirth, The stability of try-oncediscard for stochastic communication channels: Theory and validation, In 2015 54th IEEE Conference on Decision and Control (CDC). (2015) 4170-4175.

[6] W. P. M. H. Heemels, D. Nesic, A. R. Teel, N. Van de Wouw, Networked and quantized control systems with communication delays, In Proceedings of the $48 \mathrm{~h}$ IEEE Conference on Decision and Control (CDC) held jointly with 2009 28th Chinese Control Conference. (2009) 7929-7935.

[7] X. Mao, Stochastic differential equations and applications, Elsevier, 2007.

[8] D. Nesic, D. Liberzon, A unified framework for design and analysis of networked and quantized control systems, IEEE Trans. Automatic Control. 54 (2009) 732-747.

[9] P. Naghshtabrizi, J. P. Hespanha, A. R. Teel, Stability of delay impulsive systems with application to networked control systems, Transactions of the Institute of Measurement and Control. 32 (2010), 511-528.

[10] G. C. Walsh, O. Beldiman, L. G. Bushnell, Asymptotic behavior of nonlinear networked control systems, IEEE transactions on automatic control. 46 (2001) 1093-1097.

[11] G. C. Walsh, O. Beldiman, L. G. Bushnell, Error encoding algorithms for networked control systems, Automatica. 38 (2002) 261-267.

[12] G. C. Walsh, H. Ye, L. G. Bushnell, Stability analysis of networked control systems, IEEE transactions on control systems technology. 10 (2002) 438-446.

[13] L. Zhang, Y. Shi, T. Chen, B. Huang, A new method for stabilization of networked control systems with random delays, IEEE Transactions on automatic control. 50 (2005) 1177-1181. 